\title{
Sulfated Dolicholphosphate Oligosaccharides are Transiently Methylated during Biosynthesis of Halobacterial Glycoproteins*
}

\author{
JOHANN LECHNER, FELIX WIELAND, and MANFRED SUMPER \\ Institut für Biochemie I der Universität Regensburg, 8400 Regensburg, Federal Republic of Germany
}

\begin{abstract}
Summary
A novel type of N-glycoside linkage, Asn-Glc, has recently been described (Wieland et al., 1983), which occurs in the cell surface glycoprotein of the archaebacterium Halobacterium halobium. Sulfated oligosaccharides containing glucuronic acid (and, within some of the' oligosaccharides, iduronic acid) are bound via a glucose residue to Asn residues in the cell surface glycoprotein. Lipid-linked precursors of this type of glycoconjugates (Lechner et al., 1985 a) exhibit the following unusual features: The carrier lipid is a $\mathrm{C}_{60^{-}}$ dolichol rather than a bacterial undecaprenol. Sulfation of the saccharides is completed whilst they are linked to the lipid, and does not occur after transfer of the saccharides to the protein. The sulfated oligosaccharide is bound to the dolichol via a monophosphate rather than a pyrophosphate bridge. Some lipid oligosaccharides contain an additional 3-0-methylglucose residue (Lechner et al., 1985 b). This methylated sugar is peripherally linked to the lipid-saccharide intermediates and occurs exclusively at the lipid linked level, but is absent when the oligosaccharide is linked to protein. When S-adenosylmethioninedependent methylation of this glucose residue is inhibited in vivo in halobacteria, sulfated glycoprotein synthesis is depressed as well. From these data, we conclude that transient methylation of sulfated lipid oligosaccharides is a prerequisite for the transfer of the saccharide moiety to the acceptor proteins, such as the cell surface glycoprotein and the flagellins.
\end{abstract}

Key words: Glycoprotein biosynthesis - Transient methylation - Halobacteria - Dolichol - Flagellins

\section{Introduction}

Halobacteria were the first bacteria shown to posses a true glycoprotein, namely their cell surface glycoprotein (Mescher and Strominger, 1976). Subsequent work revealed that this glycoprotein exhibits some unusual features. It contains covalently linked sulfate (Wieland et al., 1980). The sulfate residues turned out to be linked to different types of saccharides: 1) to a high molecular glycoconjugate made up of a serial repeating unit containing amino sugars and hexuronic acids thus ressembling the animal glycosaminoglycans (Wieland et al., 1981/1982) and 2) to oligosaccharides containing glucuronic acid that are linked to Asn-residues of the protein via a glucose

* Paper given at the International Workshop on Biology and Biochemistry of Archaebacteria, Munich-Martinsried, June 27 to 30, 1985 .

Abbrevations: SDS gel electrophoresis: sodium dodecylsulfate polyacrylamide gel electrophoresis; SP: Sulfated precursor; GLC: gas liquid chromatography; MS: mass spectroscopy; Glc: glucose; 3-0-Me-Glc: 3-0-methylglucose; GlcUA: glucuronic acid; Ido: idose: HexUA: Hexuronic acid. residue representing a novel type of $\mathrm{N}$-glycosidic linkage in a glycoprotein (Wieland et al., 1983).

Incorportion of the sulfated repeating unit saccharide into protein could be inhibited by bacitracin (Mescher et al., 1976), indicating a sulfated lipid linked precursor which could be demonstrated by Wieland et al., 1981. This paper deals with the question how the novel N-glycosidic linkage Asn-Glc is synthesized. The most unexpected finding is that lipid oligosaccharides are transiently methylated during biosynthesis of the corresponding glycoproteins.

\section{Materials and Methods}

Most of the methods used in this study have been previously discribed (Wieland, et al., 1983; Lechner et al., 1985 a; 1985 b). As yet unpublished experimental details are given in the legends under the appropriate figures as well as in the text. 


\section{Results}

\section{Isolation and characterization of sulfated oligo- saccharides linked to protein via Asn-Glc}

A fraction of low molecular sulfated oligosaccharide peptides was obtained after gel filtration of pronase digested halobacterial cell surface glycoprotein (Wieland et al., 1982). From this fraction, which contained Glc and GlcUA in addition to sulfate and some ammino acids, we isolated a homogeneous sulfated aminoacyl saccharide composed of $1 \mathrm{Asx}, 3 \mathrm{GlcUA}, 1 \mathrm{Glc}$ and $3 \mathrm{SO}_{4}^{2-}$, indicating a N-glycosidic sugar-protein linkage. After treatment of this homogeneous sulfated aminoacylsaccharide with con- densed HF, we isolated a compound yielding, after acidic hydrolysis, Asx and Glc. This aminoacylsaccharide could be established by FAB-mass spectrocopy as well as ${ }^{1} \mathrm{H}$ NMR spectroscopy to be Asn-Glc in $\beta$-linkage (Wieland et al., 1983). Subsequently, we have investigated the structures of different isolated pronase derived sulfated glycopeptides from the halobacterial cell surface glycoprotein. They can be described with the following general structure:

Asn $(4 \rightarrow \beta 1)$ Glc $(4 \rightarrow 1)$ GlcUA $(4 \rightarrow 1)$ GlcUA $(4 \rightarrow 1)$ $\mathrm{R}$ with $\mathrm{R}=\mathrm{H}$, Glc, or GlcUA.

Using a different method of sugar hydrolysis, the occurrence of an additional hexuronic acid within these saccharides could be established. After acid methanolysis, hydrolysis of the samples in $0.5 \mathrm{~N} \mathrm{HCl}$ in methanol for
Fig. 1. Identification of iduronic acid.

A) Sulfated precursor was methanolized and the resulting methylglycosides and methylestermethylglycosides were submitted to GLC/MS as their pentafluoropropionyl derivatives (Lechner et al., 1985 a).The figure shows the total ion profile. X marks the unidentified hexuronic acid.

(As a result of the method used for derivations, each sugar is characterized by a set of peaks).

B) Sulfated precursor was methanolized, the resulting methylglycosides and methylesters hydrolyzed, reduced at $\mathrm{C}_{3}$ with sodium $\left[{ }^{1} \mathrm{H}\right]$-borohydride, repeatedly lactonized and the uronic acid lactones reduced with sodium $\left[{ }^{2} \mathrm{H}\right]$-borohydride (Lehrfeld, 1981). The sample was submitted to GLC/MS as alditolacetate derivatives as described (Lechner et al., 1985 a). The Figure shows the total ion profile.

C) Mass spectrum of the material corresponding to the peak lettered Ido in B.

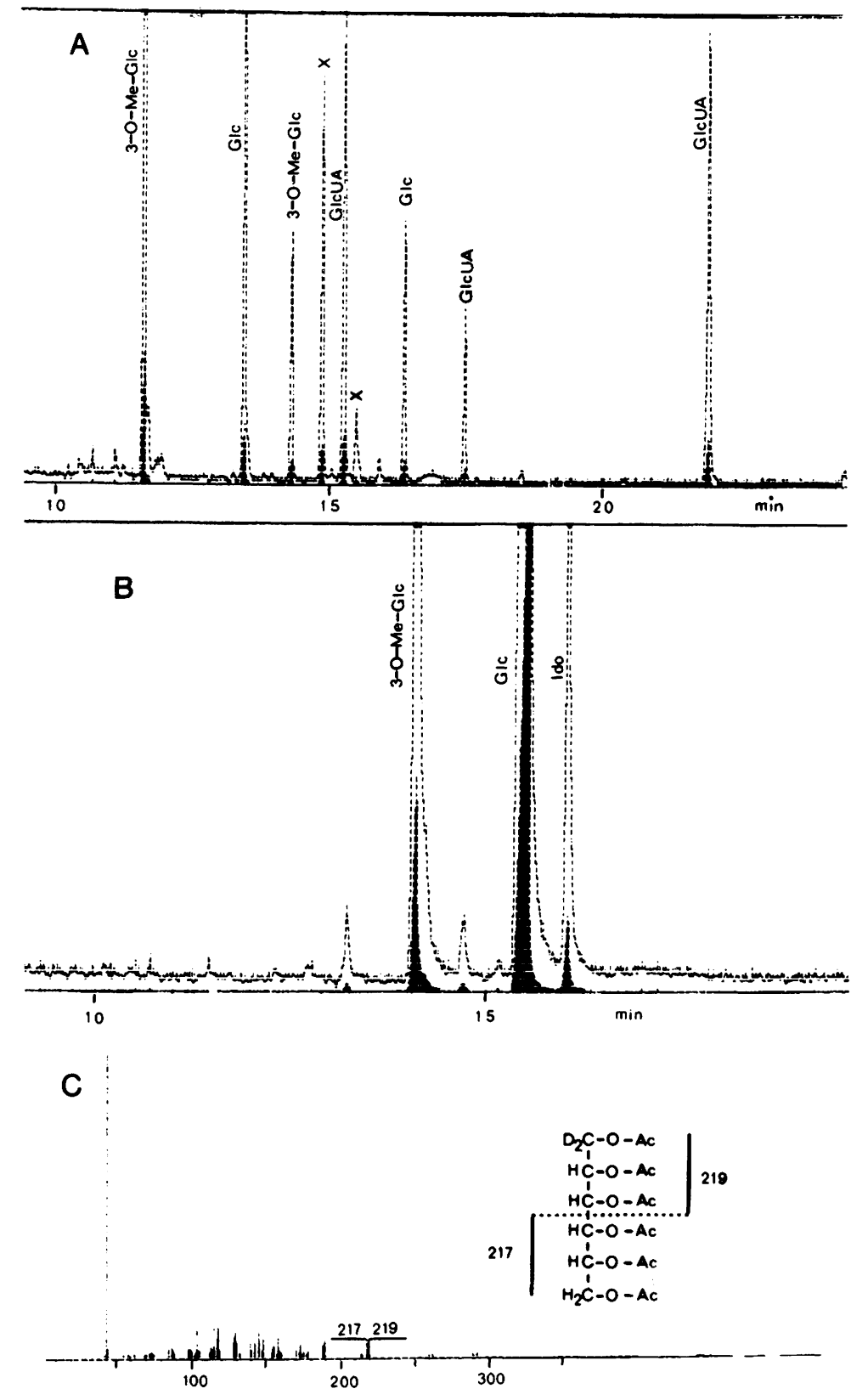


$20 \mathrm{~h}$ at $80^{\circ} \mathrm{C}$, and subsequent analysis of the corresponding pentafluoropropionylderivatives by GLC/MS, we observed an additional compound with the mass spectrum of a hexuronic acid (Fig. 1). This compound showed a retention time different from the pentafluoropropionyl derivatives of the methylestermethylglycosides of GlcUA, GalUA or ManUA. When the samples after methanolysis were hydrolyzed in $2 \mathrm{~N} \mathrm{HCl}$ for $2 \mathrm{~h}$ at $100^{\circ} \mathrm{C}$ and the resulting uronic acid lactones reduced with sodium $\left[{ }^{2} \mathrm{H}\right]$-borohydride and analyzed as their peralditol acetates by GLC/ MS, we obtained a compound eluting from the capillary column after glucose, and yielding the mass spectrum of a hexitol hexaacetate. By comparison with commercially available authentic hexoses (Sigma) this hexose was found to be idose, bearing two ${ }^{2} \mathrm{H}$ atoms at $\mathrm{C}_{6}$. Accordingly, the unknown hexuronic acid must be iduronic acid. Permethylation analyses indicated that iduronic acid represents about one third of the (1-4) linked hexuronic acids within the sulfated glycopeptides. According to preliminary results each of the hexuronic acids contains one sulfate residue in position 2 .

As mentioned above, the glycopeptides described here were isolated from the cell surface glycoprotein of halobacteria. Meanwhile we have shown that the flagellins of halobacteria, which have recently been isolated (Alam and Oesterhelt, 1984), are glycoproteins as well. The complete set of sulfated low molecular glycopeptides described above has been isolated from purified flagellar proteins (Wieland et al., 1985). Thus, halobacteria contain two types of sulfated glycoproteins: their cell surface glycoprotein with the sulfated serial repeating unit saccharide and the low molecular weight sulfated oligosaccharides, and their flagellins with identical low molecular weight sulfated saccharides.

\section{Characterization of sulfated dolicholmonophosphate oligosaccharides}

A first indication of the existence of sulfated precursors of the above mentioned olgiosaccharides had been obtained after in vivo ${ }^{35} \mathrm{SO}_{4}^{2-}$-labelling studies with subsequent SDS gel electrophoresis, as shown in Fig. 2. After short pulse labelling it is mainly a low molecular weight fraction that incorporates ${ }^{35} \mathrm{SO}_{4}^{2-}$. This sulfated material is labile. After a chase period of $120 \mathrm{~min}$ most of this radioactivity is incorporated into flagellar proteins, and, after a prolonged chase, the cell surface glycoprotein becomes markedly labeled. Therefore the labile low molecular weight material is a likely candidate to be a precursor of the sulfated glycoconjugates ( $\mathrm{SP}=$ sulfated precursor) in question. SP copurified with the halobacterial membrane fraction could be extracted into organic solvent $\mathrm{H}_{2} \mathrm{O}$-mixtures. After several steps including ion exchange chromatography SP was further purified by HPLC on Silica gel. Three main fractions of ${ }^{35} \mathrm{SO}_{4}^{2-}$-radioactivity were obtained and further analyzed. They contained lipid, phosphate, sulfate, and carbohydrate. The material was submitted to a very mild acidic condition known to cleave the labile linkage between sugar and phosphate in eucaryotic dolichylpyrophosphate oligosaccharides. The result-

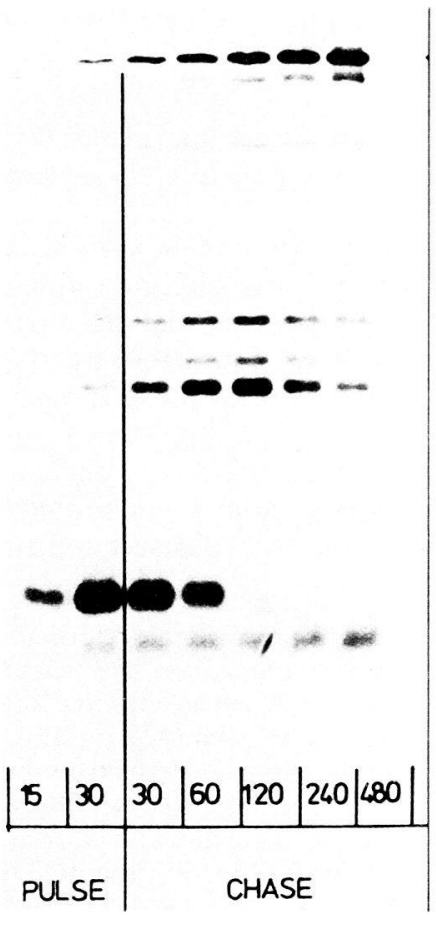

Fig. 2. Identification of a sulfated precursor of halobacterial glycoprotein. Halobacterium halobium were pulse labelled with ${ }^{35} \mathrm{SO}_{4}^{2-}$ (Lechner et al., 1985 a) and, after lysis, submitted to SDS gel electrophoresis. The figure shows the corresponding fluorogram. The array of ${ }^{35} \mathrm{SO}_{4}^{2-}$-labelled bands of middle molecular weight represents the flagellar proteins.

ing material was then partitioned between water and chloroform. Surprisingly, besides the lipid, phosphate was quantitatively found in the chloroform phase. The water phase contained carbohydrate, but no phosphate at all. After hydrolyses under conditions known to split lipid pyrophosphate into lipid phosphate and phosphate, and even much stronger acid hydrolysis conditions, all of the phosphate was found in the organic phase.

From this finding and the stoichiometry observed (1 $\mathrm{PO}_{4}^{3-}: 2 \mathrm{SO}_{4}^{2-}: 1-2 \mathrm{Glc}: 2 \mathrm{GlcUA}$ ) we must conclude that $\mathrm{SP}$ is a lipidmonophosphate oligosaccharide. EI-mass spectrocopy as well as ${ }^{1} \mathrm{H}$-NMR spectroscopy revealed that the lipid phosphate is a polyprenylphosphate of 60 carbon atoms. As allyphosphates show distinct acid lability, the failure to hydrolyze the lipid phosphate bond indicates that the phosphate-bearing isoprenyl unit must be saturated. Taken together these results show that the lipid moiety of the SP is a $\mathrm{C}_{60}$-dolichol, and that carbohydrate is linked to it via a monophosphate bridge.

The carbohydrate moiety of the three SP-fractions after HPLC were analyzed as follows: after mild treatment with methanol/HCl to specifically split the sulfuric ester bonds, the samples were reduced with sodium $\left[{ }^{3} \mathrm{H}\right]$ borohydride and chromatographed on Bio Gel P 2. Each of the HPLC fractions gave rise to 3-5 peaks after this gel filtration. The material of these individual peaks was characterized by permethylation and subsequent GLC/MS analysis. Structural details of these permethylation analyses are gi- 
Table 1. Structure of some oligosaccharides occurring linked to dolichyl-phosphate, after mild acid hydrolysis, desulphation, and reduction with sodium boronydride.

Hexuronic acids were determined to consist of about $70 \%$ glucuronic acid and $30 \%$ iduronic acid
$3-\mathrm{O}-\mathrm{CH}_{3}$-Glc $(1 \rightarrow 4) \mathrm{HexUA}(1 \rightarrow 4) \operatorname{HexUA}(1 \rightarrow 4) \mathrm{HexUA}(1 \rightarrow 4)$ Glc-ol

$3-\mathrm{O}-\mathrm{CH}_{3}$-Glc $(1 \rightarrow 4) \mathrm{HexUA}(1 \rightarrow 4) \mathrm{HexUA}(1 \rightarrow 4)$ Glc-ol

HexUA $(1 \rightarrow 4)$ HexUA $(1 \rightarrow 4) \operatorname{HexUA}(1 \rightarrow 4)$ Glc-ol

Glc $(1 \rightarrow 4)$ HexUA $(1 \rightarrow 4)$ HexUA $(1 \rightarrow 4)$ Glc-ol

Glc $(1 \rightarrow 4) \operatorname{HexUA}(1 \rightarrow 4) \operatorname{HexUA}(1 \rightarrow 4) \operatorname{HexUA}(1 \rightarrow 4)$ Glc-ol

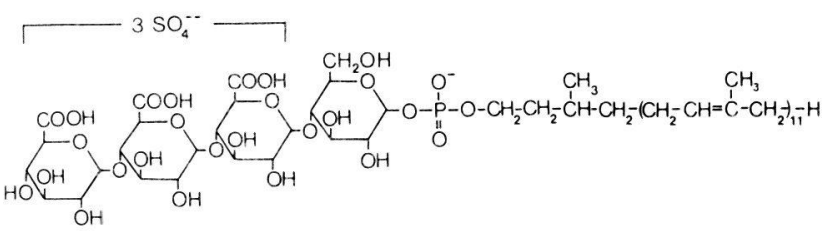

Fig. 3. Structure of one of the isolated sulfated dolicholmonophosphat oligosaccharides.

A

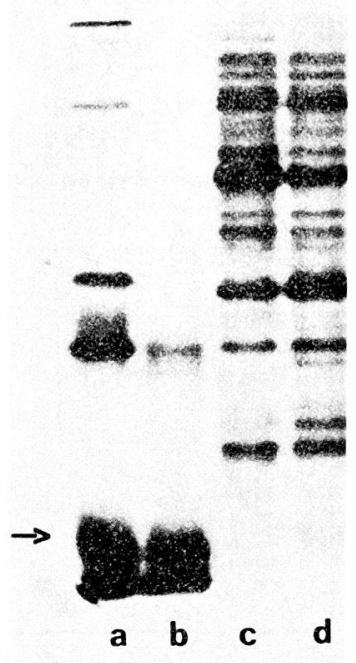

Fig. 4. Inhibition of in vivo sulfated glycoprotein biosynthesis by S-adenosyl-homocysteine, an inhibitor of S-adenosylmethionine dependent methylation.

A) Halobacterium halobium were incubated with ${ }^{35} \mathrm{SO}_{4}^{2-}$ in the absence (lane a) and presence (lane b) of $10 \mathrm{mM}$ adenosine and $10 \mathrm{mM}$ homocysteine thiolactone. Control incubations with ${ }^{35} \mathrm{~S}$-methionine in the absence (c) and presence (d) of the methylation inbitor. SDS gel electrophoresis of corresponding cell lyzates. A fluorogram of the gel is shown.

B) ${ }^{35} \mathrm{SO}_{4}^{2-}$-pulse-chase labelling of Halobacterium halobium Lane a, pulse labelling for $30 \mathrm{~min}$; lanes b-d, chase with $80 \mathrm{mM} \mathrm{Mg} \mathrm{SO}$ for 1,2 , and $3 \mathrm{~h}$, respectively; lane e, same as in a, but in the presence of $10 \mathrm{mM}$ adenosine and $10 \mathrm{mM}$ L-homocysteinethiolactone; lanes $\mathrm{f}-\mathrm{h}$ chase as above but in the presence of inhibitor of methylation. SDS gel electrophoresis of corresponding cell lyzates (Lechner et al., $1985 \mathrm{~b})$. A fluorogram of the gel is shown.

C) Quantitative evaluation of the experiment in B. Using the fluorogram as a guide, bands corresponding to the SPs (arrow in B) were cut from the gel and their radioactivity determined. 
ven in Table 1. Clearly, the carbohydrate structures of the SP fractions are compatible with those found in the sulfated glycopeptides described above. The full structure of one of the SP is given in Fig. 3.

\section{Transient methylation of the sulfated dolicholmonophosphate oligosaccharides}

Analysis of the monosaccharide constituents of some of the SPs by GLC as their peralditol accetates indicated the existence of an additional monosaccaride. This component, by GLC/MS analysis, demethylation, as well as cochromatography with an authentic compound could be elucidated to be 3-0-methylglucose. It occurs in stoichiometric amounts in two out of the three SP-fractions obtained by HPLC on silica gel, but absolutly no 3-0methylglucose could be detected neither in the cell surface glycoprotein nor in the flagellins of halobacteria. From this lack of 3-0-methylglucose in the acceptor proteins we must conclude that this residue represents a transient modification of the lipid oligosaccharides.

The following experiments were designed to investigate the role of this intermediate modification of the SP, e.g. the possible involvment in glycoprotein biosynthesis of the transient occurrence of 3-0-methylglucose.

a) Inhibition of $\mathrm{S}$-adenosylmethionine-dependent methylation inhibits sulfated glycoprotein biosynthesis. After in vivo ${ }^{35} \mathrm{SO}_{4}^{2-}$-labeling in the presence of $\mathrm{S}$ adenosylhomocysteine (an inhibitor of methylation, added as adenosine plus homocysteine-thiolactone), halobacterial cell lysates were submitted to SDS gel electrophoresis. Clearly, synthesis of the cell surface glycoprotein and of flagellins was suppressed (Fig. 4 A lanes a and b). This inhibition of glycoprotein synthesis was not due to inhibition of general protein biosynthesis, as control incubations with ${ }^{35} \mathrm{~S}$-methionine in the presence and absence of methy-

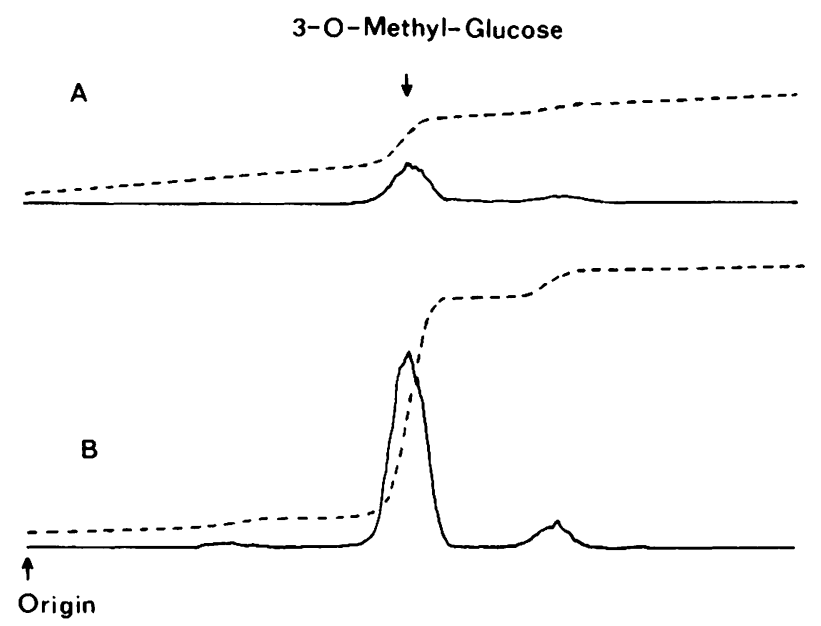

Fig. 5. Quantification of 3-0-methylglucose after labelling of the SPs with $\mathrm{CH}_{3}\left[{ }^{3} \mathrm{H}\right]$-methionine in the presence (A) and absence (B) of inhibitor of methylation.

$\mathrm{CH}_{3}-\left[{ }^{3} \mathrm{H}\right]$-labelling, and other Methods as described by Lechner et al., 1985 b. Radioscanning traces of the thin layer chromatograms are shown. The dotted line shows integration of the ${ }^{3} \mathrm{H}$ radioactivity. lation inhibitor both yielded very similar patterns of ${ }^{35} \mathrm{~S}$ methionine labelled proteins (Fig. $4 \mathrm{~A}$, lanes $\mathrm{c}$ and d). The amount of ${ }^{35} \mathrm{SO}_{4}^{2-}$-labelled SP did not seem to be decreased in the presence of S-adenosyl-homocysteine. Accordingly, in a pulse/chase experiment (Fig. $4 \mathrm{~B}$ and C), the SP fraction in the presence of inhibitor of methylation remained stable and was not incorporated into proteins.

b) To show if the observed inhibition of sulfated glycoprotein synthesis is correlated with a decrease in 3-0methylglucose containing lipid oligosaccharides, the SPfractions were isolated after in vivo labelling of halobacteria with $\mathrm{CH}_{3}-\left[{ }^{3} \mathrm{H}\right]$ methionine in the presence and $\mathrm{ab}$ sence of inhibitor of methylation. The extracted SP-fractions were then submitted to acid hydrolysis to yield monosaccharides, and the fractions of neutral sugars were purified and seperatetd by silica gel thin layer chromatography. ${ }^{3} \mathrm{H}$-radioactivity was quantitated on the chromatograms by radioscanning. As a result, the presence of S-adenosylhomocysteine had led to a decrease of 30 -methylglucose to about one fifth of the control (Fig. 5). This correlation of inhibition of the formation of 3-0methylglucose-containing lipid oligosaccharides with the

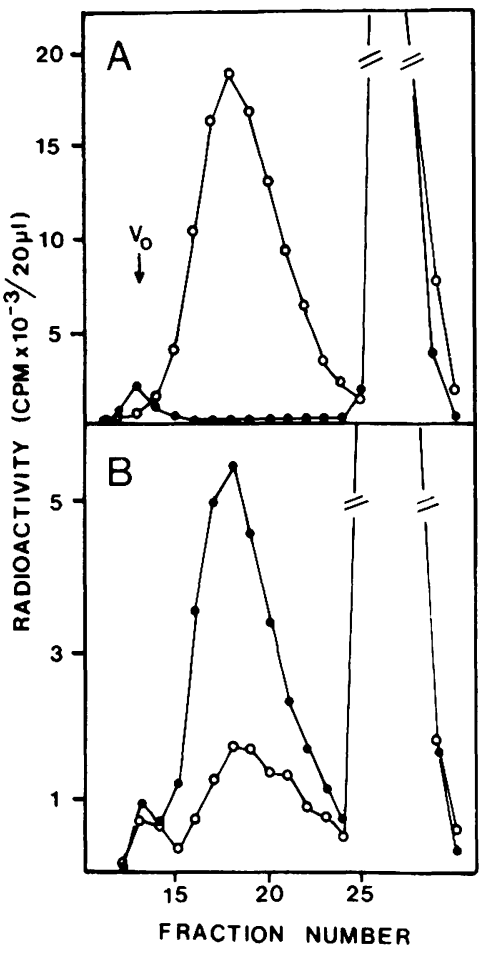

Fig. 6. Glycosylation of exogenously added acceptor peptide of the sequence Tyr-AsN-Leu-Thr-Ser-Val.

A) Black dots: Elution profile obtained after chromatography on Bio Gel P 2 of the supernatant of an incubation of $H$. halobium with ${ }^{35} \mathrm{SO}_{4}^{2-}$ in the absence of hexapeptide. Circles: same as above, but in the presence of hexapeptide as described (Lechner et al., 1985 b).

B) Black dots: Elution profile as in A of a supernatant after incubation with ${ }^{35} \mathrm{SO}_{4}^{2-}$ of the cells in the presence of hexapeptide, but without inhibitor of methylation (control). Circles: same as above, but in the presence of $10 \mathrm{mM}$ adenosine and $10 \mathrm{mM} \mathrm{L}$ homocysteinethiolactone. 
observed inhibition of sulfated glycoprotein synthesis strongly suggests that transient methylation of the sulfated lipid oligosaccharides is indeed involved in the biosynthesis of halobacterial glycoproteins.

c) The inhibition of in vivo sulfated glycoprotein biosynthesis may indicate that methylation of the sulfated dolichol oligosaccharides is necessary for the transfer to the protein of the sugar moiety. As we had obtained evidence for the occurrence of typical ASN-X-Thr(Ser)-acceptor sequences for the sulfated oligosaccharides, we used the hexapeptide Tyr-AsN-Leu-Thr-Ser-Val as an artificial exogenous acceptor to examine this possibility. Endogenous acceptor in these experiments was eliminated by the addition of ethidium bromide which leads to an immediate arrest of halobacterial protein biosynthesis. Halobacteria in sulfate-free basal salt medium were incubated with ${ }^{35} \mathrm{SO}_{4}^{2-}$ in the presence or absence of exogenous hexapeptide.

Thereafter cells were removed by centrifugation and the supernatants chromatographed on Bio Gel P 2. The radioactivity profiles obtained are shown in Fig. 6 A. Presence of the exogenous acceptor sequence hexapeptide led to the appearance of a peak a few fractions after the excluded volume. The radioactive material of this pool on high voltage thin layer electrophoresis gave a pattern very similar to that obtained by ${ }^{35} \mathrm{SO}_{4}^{2-}$-labelled pronase derived glycopeptides from the cell surface glycoprotein and the flagellins. Thus an in vivo transfering system of the sulfated oligosaccharides to an exogenous acceptor was available to investigate the influence on this transfer of the methylation inhibitor. Halobacteria were incubated with hexapeptide and ${ }^{35} \mathrm{SO}_{4}^{2-}$ in the presence or absence of $\mathrm{S}$ adenosylhomocysteine. The supernatants were again chromatographed on Bio Gel P 2. As a result, the hexapeptide-dependent radioactive peak is depressed drastically in the presence of the methylation inhibitor as shown in Fig. 6 B. Hence the observed inhibition of sulfated glycoprotein biosynthesis by $\mathrm{S}$-adenosylhomocysteine does indeed appear to be a function of inhibition of methylation of the sulfated dolichyl oligosaccharide intermediates. In addition, this result excludes any effects of the inhibitor of methylation which would somehow disturb glycoprotein core biosynthesis. The methylation seems to be involved in the passage through the membrane of the sulfated dolichol oligosaccharides and/or the transfer of the completed saccharide to protein.

\section{Discussion}

The occurrence of a $\mathrm{N}$-glycosidic bond between the $\beta$ amidonitrogen of ASN and a glucose residue represents a novel type of $\mathrm{N}$-glycosidic linkage in glycoproteins. All $\mathrm{N}$ glycosidic linkages known so far consist of $\mathrm{AsN}$ and GlcNAc. The linkage AsN-Glc is found in the cell surface glycoprotein of halobacteria as well as in their flagellins. As far as we know no other flagellins have as yet been described to be glycoproteins. The saccharides linked by this bond contain GlcUA in $(1 \rightarrow 4)$ linkage and at the nonreducing end, and some of them possess iduronic acid in $(1 \rightarrow 4)$ linkage. In addition, glucose is found at the nonreducing end of some of the saccharides. Each of the hexuronic acids present bears a sulfate residue, presumably at the 2-position. Iduronic is a main constituent of heparin, the animal glycosaminoglycans heparansulfate and dermatansulfate (Lindhal and Axelsson, 1970), and has only scarcely been detected in the kingdom of procaryotes with the exception of a cell wall polysaccharide of Chlostridium perfringens Hobbs 10 (Lee and Cherniak, 1974).

The biosynthesis of the described saccharides shows some unexpected new features:

The lipid anchor on which the saccharides are established is not of the procaryotic undecaprenol type but is a eucaryotic dolichol. The oligosaccharide is linked to this dolichol by a monophosphate bridge and not by a pyrophosphate, the linkage of all dolichyloligosaccharides investigated so far. Furthermore, the lipid monophosphate oligosaccharide is completely sulfated before transfer to protein. With the exception of the halobacterial sulfated repetitive saccharide (Wieland et al., 1982), modification of saccharides (e.g. sulfation or phosphorylation) has so far been described to occur exclusively at the protein linked level. The existence of iduronic acid within a lipid oligosaccharide has not yet been described. In the biosynthesis of the animal glycosaminoglycans, iduronic acid is described to result from epimerization of glucuronic acids at the protein linked level after completion of the saccharide chain. In some of the sulfated dolichylmonophosphate oligosaccharides, a peripherally linked 3-0-methylglucose residue has been detected. As this methylated sugar is no constituent of a biosynthetic product i. e. glycoprotein, such 3-0-methylglucose containing lipid oligosaccharides must be regarded as intermediate forms. It is not yet know whether 3-0-methylglucose is introduced from a corresponding activated sugar nucleotide or is generated by direct methylation of a peripheral glucose residue in the lipid oligosaccharide. Suppression of methylation of the lipid intermediates results in a concomitant arrest of glycoprotein synthesis. Essentially two possible functions of this methylation are conceivable. Methylation of a glucose residue in the 3-position would clearly increase the hydrophobicity of the sugar, a change resulting in a molecule with one face devoid of hydrophilic groups. Alternatively, the methylated glucose might serve as a specific recognition marker. Thus this methylation for both reasons could represent on obligatory step for translocation of the lipid oligosaccharides through the membrane. This idea is supported by our finding that an exogenous acceptor-hexapeptide is glycosylated at a much lower extent if methylation of the sulfated dolichyl oligosaccharides is inhibited.

3-0-methylglucose exists as a structural component of bacterial polysaccharides (Smith and Ballou, 1973), and, most recently, 3-0-methylmannose has been described to be a structural constituent of $\mathrm{N}$-linked oligosaccharides from Mucor rouxii (Lederkremer and Parodi, 1984), but no methylated hexose has been described so far as occurring in lipid-saccharide intermediates during glycoprotein biosynthesis. 
Halobacteria use a dolichol residue rather than a bacterial undecaprenyl residue during biosynthesis of oligosaccharide intermediates (Lechner et al., 1985 a). With respect to glycoprotein synthesis halobacteria resemble eucaryotic organisms rather than eubacteria.

In view of our data it is interesting to find out if the observed modification with a methylated sugar might play a role also in the biosynthesis of eucaryotic acidic glycoproteins or if the N-glycosidic bond Asn-Glc together with the mechanism of its biosynthesis is a speciality of archaebacteria.

Acknowledgement. This work was supported by the Deutsche Forschungsgemeinschaft '(SFB 43, Regensburg).

\section{References}

Alam, M., Oesterhelt, D.: Morphology, Function and Isolation of Halobacterial Flagella. J. molec. Biol. 176, 459-475 (1984)

Lechner, J., Wieland, F., Sumper, M.: Biosynthesis of Sulfated Saccharides N-Glycosidically linked ot the Protein via Glucose. J. Biol. Chem. 260, 860-866 (1985 a)

Lechner, J., Wieland, F., Sumper, M.: Transient Methylation of Dolichyl Oligosaccharides is an Obligatory Step in Halobacterial Sulfated Glycoprotein Biosynthesis. J. Biol. Chem. 260, 8984-8989 (1985 b)

Lederkremer, G. Z., Parodi, A. J.: 3-0-Methylation of Mannose Residues. J. Biol. Chem. 259, 12514-12518 (1984)

Lee, L., Cherniak, L.: Identification of Iduronic Acid as a Con- stituent of the "Type-Specific" Polysaccharide of Closteridium perfringens Hobbs 10. Carbohyd. Res. 33, 387-390 (1974)

Lehrfeld, J.: Differential Gas-Liquid Chromatography Method for Determination of Uronic Acids in Carbohydrate Mixtures. Analyt. Biochem. 115, 410-418 (1981)

Lindhal, U., Axelsson, O.: Identification of Iduronic Acid as the Major Sulfated Uronic Acid of Heparin. J. Biol. Chem. 246, 74-82 (1970)

Mescher, M. F., Strominger, J. L.: Purification and Characterization of a Prokaryotic Glycoprotein from the Cell Envelope of Halobacterium salinarium. J. Biol. Chem. 251, 2005-2014 (1976)

Mescher, M. F., Hansen, U., Strominger, J. L.: Formation of Lipid linked Sugar Compounds in Halobacterium salinarium. J. Biol. Chem. 251, 7289-7294 (1976)

Smith, W. L., Ballou, C. F.: The 6-0-Methylglucose-containing Lipopoly-saccharides of Mycobacterium phlei. J. Biol. Chem. 248, 7118-7125 (1973)

Wieland, F., Dompert, W., Bernhardt, G., Sumper, M.: Halobacterial Glycoprotein Saccharides Contain Covalently Linked Sulfate. FEBS Lett. 120, 110-114 (1980)

Wieland, F., Lechner, J., Bernhardt, G., Sumper, M.: Sulphation of a Repetitive Saccharide in Halobacterial Cell Wall Glycoprotein: Occurrence of a Sulphated Lipid-linked Precursor. FEBS Lett. 132, 319-323 (1981)

Wieland, F., Lechner, J., Sumper, M.: The Cell Wall Glycoprotein of Halobacteria: Structural, Functional and Biosynthetic Aspects. Zbl. Bakt. Hyg., I. Abt. Orig. C 3, 161-170 (1982)

Wieland, F., Heitzer, R., Schaefer, W.: Asparaginylglucose: Novel type of carbohydrat linkage. Proc. nat. Acad. Sci. (Wash.) 80, 5470-5474 (1983)

Wieland, F., Paul, G., Sumper, M.: Halobacterial Flagellins are Sulfated Glycoproteins. J. Biol. Chem. 260, 15180-15185 (1985)

Dr. Johann Lechner, Institut für Biochemie I der Universität, Universitätsstr. 31, D-8400 Regensburg 